\title{
Clinical and pharmacological hallmarks of rifapentine's use in diabetes patients with active and latent tuberculosis: do we know enough?
}

This article was published in the following Dove Press journal:

Drug Design, Development and Therapy

II October 2017

Number of times this article has been viewed

\author{
Chunlan Zheng',* \\ Xiufen $\mathrm{Hu}^{2}$,* \\ Li Zhao' \\ Minhui Hu' \\ Feng $\mathrm{Gao}^{3}$
}

'Department of Internal Medicine Section 5, Wuhan Pulmonary Hospital (Wuhan Tuberculosis Control Institute), ${ }^{2}$ Department of Paediatrics, Tongji Hospital, ${ }^{3}$ Department of Endocrinology, Union Hospital, Tongji Medical College, Huazhong University of Science and Technology, Wuhan, People's Republic of China

*These authors contributed equally to this work
Correspondence: Feng Gao Department of Endocrinology, Union Hospital, Tongji Medical College, Huazhong University of Science and Technology, 1277 Jiefang Avenue, Wuhan 430022, People's Republic of China Email gaofengwh@aliyun.com
Abstract: Rifapentine is a rifamycin derivate approved by the US Food and Drug Administration in 1998 for the treatment of active, drug-susceptible tuberculosis (TB). In 2014, rifapentine was approved for the treatment of latent TB infection in patients at high risk of progression to active disease and is currently under evaluation by the European Medicines Agency. Expanding indications of rifapentine largely affect diabetes patients, since about one-third of them harbor latent TB. Clinical consequences of rifapentine use in this population and potentially harmful interactions with hypoglycemic agents are widely underexplored and generally considered similar to the ones of rifampicin. Indeed, rifapentine too may decrease blood levels of many oral antidiabetics and compete with them for protein-binding sites and/or transporters. However, the two drugs differ in protein-binding degree, the magnitude of cytochrome P450 induction and auto-induction, the degree of renal elimination, and so on. Rifapentine seems to be more suitable for use in diabetes patients with renal impairment, owing to the fact that it does not cause renal toxicity, and it is eliminated via kidneys in smaller proportions than rifampicin. On the other hand, there are no data related to rifapentine use in patients $>65$ years, and hypoalbuminemia associated with diabetic kidney disease may affect a free fraction of rifapentine to a greater extent than that of rifampicin. Until more pharmacokinetic information and information on the safety of rifapentine use in diabetic patients and drug-drug interactions are available, diabetes in TB patients treated with rifapentine should be managed with insulin analogs, and glucose and rifapentine plasma levels should be closely monitored.

Keywords: rifamycin, antituberculosis treatment, glucose intolerance, hyperglycemia, safety

\section{Introduction}

In recent years, a large body of evidence that diabetes mellitus (DM) affects the natural history of tuberculosis (TB) has emerged. ${ }^{1-4}$ DM triples the risk of developing active TB, and $15 \%-25 \%$ of active TB cases globally are attributable to this disease. ${ }^{5,6}$ Additionally, according to a recent meta-analysis conducted by Lee et al, there is also a small, but the statistically significant positive causal association between DM and latent TB infection (LTBI) (adjusted OR 1.18, 95\% CI: 1.07-1.29 across 12 crosssectional studies). ${ }^{7}$

After almost half a century, anti-TB drug development is emerging again, mainly through expanding indications and reengineering of existing anti-TB compounds and discovery of new compounds. ${ }^{8}$ However, the anti-TB treatment in diabetes patients remains an issue of great complexity for several disquieting reasons: first, both DM and active TB infection may affect the pharmacokinetics of drugs (owing to changes in subcutaneous adipose tissue and muscle blood flow, nonenzymatic albumin glycation, 
renal and hepatic impairment, etc.), ${ }^{9}$ possibly lessening their efficacy (impaired bactericidal activity of anti-TB drugs or hyperglycemia due to noneffectiveness of antidiabetics) or causing toxicity; ${ }^{10}$ second, as evidenced in in vivo and in vitro studies, DM affects the innate and adaptive immune responses necessary to control $\mathrm{TB}$ infection, leading to higher rates of treatment failure, death, and relapse of TB in diabetes patients as compared to nondiabetes ones, ${ }^{11}$ third, commonly used anti-TB drugs are inhibitors (isoniazid) or inducers (rifampicin) of the cytochrome (CYP) P450 which can provoke changes in the plasma concentrations of hypoglycemic and other anti-TB drugs. ${ }^{12,13}$

Lately, several novel pipelines to anti-TB treatment have been introduced: bedaquiline and delamanid, novel anti-TB agents approved by the US Food and Drug Administration (FDA) in 2012 and the European Medicines Agency in 2014, respectively, for the treatment of multidrug-resistant TB (MDR-TB); ${ }^{14,15}$ clofazimine, linezolid, and carbapenems, repurposed drugs showing promising results in MDR- and extensively drug-resistant-TB cases; ${ }^{16}$ and a number of newly proposed regimens. ${ }^{17}$ While the focus is placed on the development of anti-MDR-TB therapies, drug-sensitive anti-TB treatment research, not surprisingly, progresses at a much slower rate and often lacks attention of scientific community. ${ }^{16,17}$

Rifapentine is an antimicrobial rifamycin derivative and has a similar profile of microbiological activity to rifampicin. ${ }^{18}$ It was initially approved by the FDA in 1998 for treatment of active, drug-sensitive TB caused by Mycobacterium tuberculosis in adults and children aged $\geq 12$ years. In 2014, the FDA approved the combination of rifapentine and isoniazid for the treatment of LTBI in patients aged $\geq 2$ years at high risk of progression to TB disease. ${ }^{18}$ This regimen is included in the WHO's first-ever Guidelines on the Management of Latent Tuberculosis Infection, released in 2015. ${ }^{19}$ In the People's Republic of China, rifapentine has been widely used in clinical settings since the 1996 version of the Chinese National Essential Medicine List was published, and there has been a growing tendency for clinicians to use rifapentine instead of rifampicin. ${ }^{20}$ Rifapentine is a potent inducer of several CYP450 enzymes and may influence the kinetics of many drugs at different levels. It may reduce the hypoglycemic effects of hypoglycemic agents and other drugs, and its use in special populations is widely unexplored. ${ }^{18}$ While the potential for these effects is largely unknown for rifapentine, rifampicin, also a potent inducer of CYP450, was found to enhance the glucose-lowering action of metformin and decrease the plasma concentrations of nateglinide and glyburide. ${ }^{21-23}$ Knowing that around $25 \%$ of diabetes patients harbor LTBI and that DM triples the risk of active TB, ${ }^{5,24}$ the expanding use of rifapentine creates a need for clarifying the efficacy and safety profile of this drug when it comes to a vulnerable population such as diabetes patients. Therefore, in this paper, we aimed to review all clinical and pharmacological data relevant for the concurrent use of rifapentine and oral hypoglycemic agents/insulin, to compare them with those of rifampicin, and to potentially identify optimal combinations of rifapentine and hypoglycemic drugs in terms of patient's safety.

\section{Search methodology}

We searched PubMed/MEDLINE, EMBASE, Google Scholar, and Scopus for studies of all designs using the following key words: "hypoglycemic", "antidiabetic", "glucose-lowering", "rifapentine", "rifampicin", and "rifamycin" without applying any language or other restrictive criteria. The WHO Guidelines on the Management of Latent Tuberculosis Infection and Summaries of Product Characteristics (SmPCs) of all drugs mentioned in this review were referred to obtain the most actualized data on the management of latent TB and additional information regarding safety profiles of the included drugs.

\section{Pharmacokinetic hallmarks of rifapentine and possible interactions with hypoglycemic agents Pharmacokinetics of rifapentine}

While pharmacokinetic and pharmacodynamic properties of rifampicin and their clinical significance are widely known, ${ }^{13}$ clinical repercussions of pharmacological differences between rifampicin and rifapentine are largely unexplored.

In the initial phase of active TB ( 2 months), rifapentine tablets should be administered at a dose of $600 \mathrm{mg}$ twice weekly for 2 months as directly observed therapy (DOT), with an interval of no less than $72 \mathrm{~h}$ between doses, with daily administration of isoniazid, ethambutol, or pyrazinamide. In the continuation phase ( 4 months), the drug should be given at a dose of $600 \mathrm{mg}$ once per week with DOT of isoniazid or an appropriate agent for susceptible organisms. ${ }^{18}$ For LTBI, the dose of rifapentine should be determined based on the weight of the patient (up to a maximum of $900 \mathrm{mg}$ once weekly for 12 weeks), and the drug should be accompanied by isoniazid. ${ }^{18}$ The absolute bioavailability of rifapentine after a single $600 \mathrm{mg}$ dose in healthy adult volunteers is $70 \% .{ }^{18,25}$ Unlike rifampicin that shows supraproportional increase in exposure with increasing doses, ${ }^{26}$ increases in 
the dose of rifapentine result in less-than-dose-proportional increases in exposures. ${ }^{27}$ Similarly, while absorption of rifampicin decreases when taken with food, ${ }^{13}$ administration of rifapentine with a high fat meal ( 850 total calories) increases the area under the curve (AUC) $(0-\infty)$ and maximal concentration (Cmax) by $43 \%$ and $44 \%$, respectively, over that observed when administered under fasting conditions. ${ }^{18,25}$ As recently shown by Savic et al, although the bioavailability of rifapentine decreased with increasing dose as compared with the lowest dose administered (450 mg), high exposures could be achieved when rifapentine was given daily at high doses with food. In this study, target rifapentine AUC0-24 $\geq 350 \mu \mathrm{g} \cdot \mathrm{h} / \mathrm{mL}$ (AUC95) was achieved with a daily dose of $1,200 \mathrm{mg}$ in $\geq 87 \%$ of participants who took the drug with high-fat foods, and when compared with control group treated with rifampicin, these patients were estimated to take 3.7 weeks less to develop stable culture conversion to negative in liquid media. ${ }^{28}$ While both rifampicin and rifapentine are substrates for p-glycoprotein (ABCB1), it seems that only rifampicin has the potential to induce it. ${ }^{13,18,25,29}$ The Cmax is normally achieved from 5 to $6 \mathrm{~h}$ after administration of the $600 \mathrm{mg}$ dose of rifapentine. ${ }^{18}$ In healthy volunteers, rifapentine and its active metabolite, 25-desacetyl rifapentine, are $99 \%$ and $93.2 \%$ bound to plasma proteins, respectively, mainly to albumin. ${ }^{18}$ Rifampicin protein binding is about $80 \%$, but the clinical impact of higher protein binding of rifapentine remains unclear. ${ }^{13}$ Both drugs are deacetylated by liver esterase. ${ }^{25,29}$ Rifapentine has a much longer half-life than rifampicin (10-15 h compared to $2-5 \mathrm{~h}$, respectively). Around $15 \%$ of rifapentine is eliminated by urine, whereas in case of rifampicin, renal elimination reaches $30 \%{ }^{13,18,25,29}$

Similar to rifampicin, rifapentine induces microsomal enzymes of the liver, mainly CYP3A4 and CYP2C8/9, and therefore accelerates the metabolism of some drugs. ${ }^{18,25}$ Induction of enzyme activities occurs within 4 days after taking the first dose of rifapentine and within 2 days after taking the first dose of rifampicin, and enzyme activities return to baseline levels 14 days after discontinuing both drugs. ${ }^{13,18,25,29}$ The magnitude of enzyme induction of rifapentine is smaller than the one of rifampicin. ${ }^{25,29}$ However, while rifampicin has a strong potential of auto-induction, rifapentine shows no such effect. ${ }^{25,29}$ The SmPCs of rifampicin and rifapentine provide a list of drugs, the concomitant use of which should be monitored. Among antidiabetic agents, only sulfonylureas are mentioned as potentially dangerous when combined with the rifamycin group. ${ }^{13,18}$ Pharmacokinetic characteristics of rifapentine and rifampicin after oral administration are shown in Table 1.
Table I Pharmacokinetic characteristics of rifapentine and rifampicin after oral administration

\begin{tabular}{lll}
\hline PK properties & Rifapentine & Rifampicin \\
\hline Intestinal absorption & Increases with food & Decreases with food \\
Substrate of ABCBI & Yes & Yes \\
Inducer of ABCBI & No & Yes \\
Bioavailability after & $\sim 70$ & $\sim 90$ \\
administration per os (\%) & & \\
C $_{\max }$ (h) & $2.5-5.5$ & $2-4$ \\
Plasma protein binding (\%) & $\sim 99$ & $\sim 80$ \\
Biotransformation & Hepatic esterase & Hepatic esterase \\
CYP450 induction & $3 \mathrm{A4}, 2$ C8/9 & IA2, 3A4, 2C8/9/19 \\
Magnitude of CYP450 & ++ & +++ \\
induction & & \\
Auto-induction & No & Yes \\
Half-life (h) & $\sim 10-15$ & $\sim 2-5$ \\
Main elimination route & Hepatic & Hepatic \\
Renal elimination (\%) & $\sim 15$ & $\sim 30$ \\
\hline
\end{tabular}

Abbreviations: PK, pharmacokinetic; $C_{\text {max }}$ maximal concentration; CYP, cytochrome P.

\section{Interactions between rifapentine and oral hypoglycemic agents}

The use of rifapentine in diabetes patients has not been studied, although studies suggest that exposure to rifampicin is strongly reduced in patients with $\mathrm{TB}$ and $\mathrm{DM}$ as compared to nondiabetic TB patients..$^{30}$ In spite of the pharmacological differences between rifampicin and rifapentine, and the very likely potential for pharmacokinetic drug-drug interactions of rifapentine, surprisingly small number of studies performed on humans addressed this topic. ${ }^{30-34}$ Although it has been shown that the magnitude of enzyme induction of rifapentine is smaller than that of rifampicin, ${ }^{25,29}$ the study of Dooley et al found that the mean AUC0-12 of oral midazolam, a CYP3A probe drug, was reduced by $93 \%$ with the coadministration of rifapentine and by $74 \%$ with the coadministration of rifampicin $(p<0.01) .{ }^{35}$ Therefore, the potential for drug interaction of the two drugs may not be entirely comparable.

The parallel use of rifapentine and sulfonylureas is recognized as potentially dangerous in terms of lack of efficacy of the hypoglycemic agents due to induction of CYP3A4. ${ }^{18}$ However, this type of interaction may be expected with many other oral antidiabetics, like repaglinide, pioglitazone, glibenclamide, sitagliptin, bromocriptine mesylate, and others, which also use this metabolic pathway. By inducing $\mathrm{CYP} 2 \mathrm{C} 8$, rifapentine may decrease serum concentrations of gliclazide and rosiglitazone, and additionally modulate the kinetics of pioglitazone and sitagliptin. ${ }^{36}$ In the study of Zheng et al, multiple oral rifampicin doses lowered glyburide's AUC and Cmax by $63 \%$ and $48 \%$, respectively, and 
increased blood glucose levels compared to control. ${ }^{37}$ The fact that both drugs share the potential to induce CYP3A4 and CYP2C8/9 leads to the conclusion that the effect observed by Zheng et al may indeed be expected in case of rifapentine as well. However, it is important to take into account that the effect of rifampicin on AUC and Cmax of glyburide is probably due to both p-glycoprotein and CYP3A4 induction, and OATP1B1 inhibition, which does not make the two drugs entirely comparable. In vitro studies suggest that, unlike rifampicin, rifapentine does not induce p-glycoprotein. ${ }^{25,29}$ Nevertheless, since this transporter becomes saturated by high concentrations of drug in the intestinal lumen, rifapentine may compete with drugs that are also substrate of this transporter, like sulfonylureas and/or SGLT-2 agents. ${ }^{38}$ Since sulfonylureas are also largely affected by the CYP3A4 or CYP2C8 induction, ${ }^{36}$ this may be of particular clinical importance. Crossed metabolic pathways between different oral antidiabetics and rifapentine/rifampicin are shown in Table 2.

Interferons and pro-inflammatory cytokines can downregulate CYP450 expression in vivo and in hepatocyte cultures, leading to an effect opposite to the one observed with rifamycin agents - decreased metabolism of oral antidiabetic drugs. ${ }^{39-41}$ Therefore, pharmacokinetic studies comparing diabetes patients with TB on different antidiabetic regimens with nondiabetes $\mathrm{TB}$ patients must be performed to explain the clinical significance of the opposite effects that rifamycin drugs and infection/inflammation have on CYP450 expression.

Rifapentine is highly bound ( $\sim 99 \%$ ) to plasma proteins; ${ }^{18}$ hence, coadministered drugs may compete for the same

Table 2 Crossed metabolic pathways of rifapentine/rifampicin and oral antidiabetics

\begin{tabular}{|c|c|c|}
\hline \multirow[t]{2}{*}{ Oral hypoglycemic agent } & \multicolumn{2}{|c|}{ Antituberculosis drug } \\
\hline & Rifapentine & Rifampicin \\
\hline \multicolumn{3}{|l|}{ Meglitinides } \\
\hline Nateglinide $\left(\right.$ Starlix $\left.{ }^{\circledR}\right)$ & $3 A 4 / 2 C 9$ & $3 \mathrm{~A} 4 / 2 \mathrm{C} 9$ \\
\hline Repaglinide (Prandin $\left.{ }^{\circledR}\right)$ & $3 \mathrm{~A} 4 / 2 \mathrm{C} 8$ & $3 \mathrm{~A} 4 / 2 \mathrm{C} 8$ \\
\hline \multicolumn{3}{|l|}{ Thiazolidinediones } \\
\hline Pioglitazone $\left(\right.$ Actos $\left.^{\circledR}\right)$ & $3 \mathrm{~A} 4 / 2 \mathrm{C} 8$ & $3 \mathrm{~A} 4 / 2 \mathrm{C} 8$ \\
\hline Rosiglitazone $\left(\right.$ Avandia $^{\circledR}$ ) & $2 C 8 / 9$ & $2 C 8 / 9$ \\
\hline \multicolumn{3}{|l|}{ Sulfonylureas } \\
\hline Glibenclamide (Glyburide ${ }^{\circledR}$ ) & $3 \mathrm{~A} 4$ & $3 \mathrm{~A} 4$ \\
\hline Gliclazide (Diamicron ${ }^{\circledR}$ ) & $2 C 8 / 9$ & $2 C 8 / 9 / 19$ \\
\hline Gliquidone (Glurenorm ${ }^{\circledR}$ ) & $3 \mathrm{~A} 4 / 2 \mathrm{C} 9$ & $3 \mathrm{~A} 4 / 2 \mathrm{C} 9 / 19 / 1 \mathrm{~A} 2$ \\
\hline \multicolumn{3}{|l|}{ DPP-IV inhibitors } \\
\hline Sitagliptin (Januvia $\left.{ }^{\circledR}\right)$ & $3 \mathrm{~A} 4 / 2 \mathrm{C} 8$ & $3 \mathrm{~A} 4 / 2 \mathrm{C} 8$ \\
\hline Saxagliptin (Onglyza ${ }^{\circledR}$ ) & $3 \mathrm{~A} 4$ & $3 \mathrm{~A} 4$ \\
\hline \multicolumn{3}{|l|}{ Other } \\
\hline Bromocriptine mesylate $\left(\right.$ Cycloset $\left.^{\circledR}\right)$ & $3 \mathrm{~A} 4$ & $3 \mathrm{~A} 4$ \\
\hline
\end{tabular}

Abbreviation: DPP-IV, dipeptidyl peptidase IV. plasma protein-binding sites and affect the free drug concentration. This may be the case with their concurrent use with sulfonylureas (protein binding $>99 \%$ ), glinides (protein binding $>98 \%$ ), or SGLT-2 inhibitors (protein binding $>98 \%$ ). ${ }^{36}$ The pharmacokinetic and clinical consequences of potential protein-binding displacement remain to be elucidated.

Metformin suppresses the inflammatory response by nuclear factor $\kappa \mathrm{B}$ inhibition via adenosine monophosphateactivated protein kinase-dependent and kinase-independent pathways, and its anti-inflammatory properties are independent of patient's DM status. ${ }^{42}$ Also, it does not use the CYP450 metabolic pathway, its transport is p-glycoproteinindependent, and it does not bind to plasma proteins. ${ }^{43}$ For its favorable pharmacokinetic and anti-inflammatory profile, metformin could be a key compound for combination with rifamycin drugs. However, Cho et al found that rifampicin enhances the glucose-lowering effect of metformin and increases the OCT1 mRNA levels in healthy participants. ${ }^{21}$ Williamson et al showed that rifapentine does not affect the expression of ABCB1, ABCC2, OATP1B1, or OATP1B3, ${ }^{44}$ but currently no preclinical or clinical studies explain its influence on OCTs.

\section{Interactions between rifapentine and insulin analogs}

The hyperglycemia associated with TB may complicate the management of diabetes. ${ }^{45}$ An optimal glycemic control results in a better patient outcome, so management of DM in TB should be aggressive. ${ }^{45}$ The American Association of Clinical Endocrinologists discourages the use of traditional human insulins, recommending modern insulins or insulin analogs instead, as they are more predictable in action and cause less hypoglycaemia. ${ }^{46}$ A limited amount of exogenous insulin is metabolized by the liver in its first pass; the main organ responsible for metabolizing exogenous insulin administered to diabetes patients $(\sim 80 \%)$ is the kidney. Approximately $65 \%$ of insulin is filtered in the glomerulus and metabolized in the cells of the proximal tubule. The remaining insulin $(\sim 35 \%)$ diffuses from post-glomerular peritubular vessels to the contraluminal cell membrane of the proximal tubular cell, where it is also degraded. ${ }^{47-50}$ Although insulin analogs are also metabolized in the liver, ${ }^{51}$ they do not share metabolic pathways with rifapentine, so the hepatic alteration of the pharmacokinetic parameters of these drugs is not expected.

Although the SmPCs of the insulin analogs do not include specific drug interactions, it is generally thought that several drugs potentially increase the hypoglycemic 
effect of insulin (oral antidiabetic products, pramlintide, angiotensin-converting enzyme inhibitors, fibrates, fluoxetine, mono-amine oxidase inhibitors, propoxyphene, pentoxifylline, salicylates, somatostatin analogs, sulfonamide antibiotics) and reduce it (corticosteroids, niacin, diuretics, sympathomimetic agents, glucagon, isoniazid, phenothiazine derivatives, thyroid hormones, estrogens, progestogens, protease inhibitors, atypical antipsychotic medications). ${ }^{52-57}$ Nevertheless, currently, there are no data related to potential hypoglycemic or hyperglycemic properties of rifapentine, which may affect the hypoglycemic potential of insulin analogs.

Following subcutaneous injection, insulin detemir and degludec extensively bind to albumin. The prolonged action of detemir and degludec, achieved by slow absorption from the subcutaneous depot, appears to be partially mediated by binding to albumin via a fatty acid chain. ${ }^{56,57}$ Clinical data on the relationship between the pharmacodynamic and pharmacokinetic properties of detemir and degludec, and the plasma level of albumin are very scarce. However, due to their high-level protein-binding, potential interactions of detemir and degludec with rifapentine should be considered.

\section{Interactions between rifapentine and GLP-I receptor agonists/pramlintide}

Liraglutide is the only drug from this group which may possibly interact with rifapentine at the protein-binding level, as it is highly protein-bound $(>98 \%) .{ }^{58}$ Pramlintide is metabolized primarily by the kidneys, while the GLP-1 receptor agonists undergo minimal systemic metabolism or they are endogenously metabolized by dipeptidyl peptidase IV and endogenous endopeptidases. ${ }^{59}$ Hence, other pharmacokinetic interactions are not expected with rifapentine. Slower gastric emptying induced by GLP-1 receptor agonists may reduce the extent and rate of absorption of both rifapentine and rifampicin and affect their efficacy, as it at least partially depends on the blood concentrations of drugs. ${ }^{25,29,59}$ Possible pharmacokinetic interactions between rifapentine/rifampicin and hypoglycemic agents and their expected clinical effects are shown in Table 3.

\section{Pharmacodynamic hallmarks of rifapentine and possible interactions with hypoglycemic agents Mechanism of action and pharmacodynamic interactions of rifapentine}

Rifapentine inhibits DNA-dependent RNA polymerase in susceptible strains of both intracellular and extracellular M. tuberculosis organisms, but not in mammalian cells. ${ }^{18,25}$ Rifapentine and the 25-desacetyl metabolite accumulate in human monocyte-derived macrophages with intracellular/ extracellular ratios of approximately $24: 1$ and 7:1, respectively. Interpretive minimal inhibitory concentration (MIC) criteria/

Table 3 Possible PK interactions between rifapentine/rifampicin and hypoglycemic agents and their expected clinical effects

\begin{tabular}{|c|c|c|}
\hline Interaction level & Possible PK interaction & Expected clinical effect \\
\hline $\begin{array}{l}\text { Transporter level } \\
(\mathrm{ABCBI})\end{array}$ & $\begin{array}{l}\text { Rifapentine: competition with SU and SGLT-2 inhibitors. Decreased levels of SU } \\
\text { and SGLT-2 inhibitors } \\
\text { Rifampicin: competition with SU and SGLT-2 inhibitors and induction of ABCBI. } \\
\text { Decreased levels of SU and SGLT-2 inhibitors }\end{array}$ & $\begin{array}{l}\text { Lack of hypoglycemic efficacy, possibly greater } \\
\text { with rifampicin }\end{array}$ \\
\hline $\begin{array}{l}\text { Protein-binding } \\
\text { level }\end{array}$ & $\begin{array}{l}\text { Rifapentine: competition for protein-binding sites with SU, glinides, SGLT-2 } \\
\text { inhibitors, detemir, degludec, and liraglutide. Increased levels of oral antidiabetic } \\
\text { drugs and/or rifapentine } \\
\text { Rifampicin: competition for protein-binding sites with SU, glinides, SGLT-2 } \\
\text { inhibitors, detemir, degludec, and liraglutide. Increased levels of oral antidiabetic } \\
\text { drugs and/or rifampicin }\end{array}$ & $\begin{array}{l}\text { Possible potentiation of hypoglycemic } \\
\text { and/or antituberculosis effects, and increased } \\
\text { risk of dose-dependent adverse effects. } \\
\text { The interaction may be stronger with } \\
\text { rifapentine than with rifampicin }\end{array}$ \\
\hline \multirow[t]{4}{*}{$\begin{array}{l}\text { Hepatic } \\
\text { metabolism level }\end{array}$} & $\begin{array}{l}\text { Rifapentine: induction of CYP3A4 and CYP2C8/9 and decreased levels of } \\
\text { nateglinide, repaglinide, pioglitazone, rosiglitazone, glibenclamide, gliquidone, } \\
\text { gliclazide, sitagliptin, and saxagliptin }\end{array}$ & Rifapentine: hyperglycemia \\
\hline & $\begin{array}{l}\text { Rifampicin: induction of CYP3A4, CYP2C8/9/19, CYPIA2, and auto-induction. } \\
\text { Decreased levels of nateglinide, repaglinide, pioglitazone, rosiglitazone, } \\
\text { glibenclamide, gliquidone, gliclazide, sitagliptin, saxagliptin, and rifampicin itself }\end{array}$ & $\begin{array}{l}\text { Rifampicin: hyperglycemia and diminished } \\
\text { antituberculosis efficacy over time }\end{array}$ \\
\hline & $\begin{array}{l}\text { Rifapentine/rifampicin: induction by antidiabetics with CYP3A4-inducing } \\
\text { potential, like bromocriptine. Decreased levels of both rifapentine and rifampicin }\end{array}$ & $\begin{array}{l}\text { Lack of antituberculosis efficacy, possibly } \\
\text { greater with rifampicin owing to auto-induction }\end{array}$ \\
\hline & $\begin{array}{l}\text { Rifapentine/rifampicin/antidiabetics: inhibition of CYP450 in severe infection. } \\
\text { Increased levels of oral antidiabetic drugs and/or rifapentine/rifampicin }\end{array}$ & $\begin{array}{l}\text { Possible potentiation of hypoglycemic and/or } \\
\text { antituberculosis effects, and increased risk of } \\
\text { dose-dependent adverse effects }\end{array}$ \\
\hline
\end{tabular}

Abbreviations: PK, pharmacokinetic; SU, sulfonylureas; CYP, cytochrome P. 
breakpoints to determine the susceptibility of $M$. tuberculosis to rifapentine have not been established. ${ }^{18,25}$ However, in vitro preclinical studies showed that $M$. tuberculosis complex strains are more susceptible to rifapentine than to rifampicin, irrespective of the testing method. ${ }^{60}$

Clinical studies exploring the effect of DM on the pharmacodynamics of drugs are very scarce. Several studies have reported the effects of DM on the drug dose response of certain cardiovascular drugs, ${ }^{61-63}$ but it is unclear whether these studies show true pharmacodynamic changes or merely pharmacokinetic changes.

Clinical studies performed with rifapentine did not specifically address the dose/concentration-effect relationship between rifapentine and other drugs at the receptor, signaling, or effector level. However, both rifapentine and rifampicin may provoke alterations in glucose metabolism (hypo- and hyperglycemia $)^{13,18}$ and potentially interfere with the hypoglycemic effect of antidiabetic drugs. Therefore, combining these drugs requires caution.

\section{Mechanisms of resistance}

The incidence of rifapentine-resistant organisms associated with spontaneous mutations in an otherwise susceptible population of $M$. tuberculosis strains is approximately one in $10^{7}-10^{8}$ bacilli. $^{25}$ Although there is evidence of complete cross-resistance between rifampicin and rifapentine, ${ }^{64}$ some authors demonstrated that rifampicin-resistant strains still preserved susceptibility to rifapentine. ${ }^{65}$ Nevertheless, resistance to both rifapentine and rifampicin normally develops quickly when exposed to a single drug, ${ }^{13,18}$ so they should only be used in combination with other drugs when treating active TB. Single-base-pair mutations in the $\beta$ subunit of the RNA polymerase gene $(r p o B)$ are responsible for the development of cross-resistance to all rifamycin drugs. ${ }^{64}$

\section{Clinical efficacy and safety}

Rifapentine was studied in two randomized, open-label controlled clinical trials in the treatment of active pulmonary TB and one multicenter, open-label, randomized, activecontrolled trial in latent pulmonary TB. The first trial included 722 HIV-negative patients with active pulmonary TB treated with rifapentine $600 \mathrm{mg}$ twice a week in combination with daily isoniazid, pyrazinamide, and ethambutol $(n=361)$ and rifampicin $600 \mathrm{mg}$ in the same combination administered daily in the initial 2-month phase of treatment. ${ }^{18}$ During the 4-month continuation phase, 317 patients in the rifapentine group continued to receive rifapentine $600 \mathrm{mg}$ dose once weekly with isoniazid and 304 patients in the rifampicin group received rifampicin and isoniazid twice weekly. ${ }^{18}$ At the end of 6 months of treatment, more patients from rifapentine group reached conversion ( $87 \%$ from rifapentine compared to $80 \%$ from rifampicin group). However, through 24-month follow-up period, the risk of relapse was greater in the group treated with the rifapentine combination. ${ }^{18}$ The second trial was performed in 1,075 HIV-negative and HIV-positive patients with active pulmonary TB who had completed the initial 2-month phase of treatment with rifampicin, isoniazid, pyrazinamide, and either ethambutol or streptomycin. The patients were randomly assigned to receive either rifapentine $600 \mathrm{mg}$ and isoniazid $15 \mathrm{mg} / \mathrm{kg}$ once weekly (502 HIV-negative and 36 HIV-positive patients) or rifampicin $10 \mathrm{mg} / \mathrm{kg}$ and isoniazid $15 \mathrm{mg} / \mathrm{kg}$ twice weekly (502 HIV-negative and $35 \mathrm{HIV}$-positive patients) for the 4-month continuation phase. Around $94 \%$ of patients from rifapentine group reached conversion after 6 months of therapy compared to $91 \%$ from rifampicin group. Both HIVpositive and HIV-negative patients had higher rates of relapse with rifapentine than with rifampicin after 24 months. ${ }^{18}$

The effectiveness of 12 weekly doses of rifapentine in combination with isoniazid $(n=3,074)$ compared to 9 months of self-administered daily isoniazid $(n=3,074)$ was studied in patients with positive tuberculin skin test and at high risk of progression to TB disease. Active TB developed in 5 of 3,074 randomized patients in the rifapentine + isoniazid group $(0.16 \%)$ versus 10 of 3,074 patients in isoniazid-only group $(0.32 \%)$, for a difference in cumulative rates of $0.17 \%$ (95\% CI: -0.43 to 0.09$).{ }^{18}$

Most frequently reported side effects of rifapentine $(>1 \%$ of patients) were hyperuricemia (most likely due to pyrazinamide from the initiation phase), pyuria, hematuria, urinary tract infection, neutropenia, lymphocytopenia, anemia, chest pain, edema, headache, diaphoresis, skin rash, hypoglycemia, hyperglycemia, anorexia, nausea and vomiting, constipation, dyspepsia, abdominal pain, diarrhea, hypertransaminasemia, arthralgia, hemoptysis, and cough. ${ }^{18}$

\section{Clinical considerations related to the use of rifapentine in diabetes patients \\ Aging}

Patients affected by type 2 DM are predominantly over 60 years of age. Aging is associated with structural and functional changes that affect the metabolism and pharmacodynamics of drugs. ${ }^{66,67}$ Thus, the therapeutic effects and safety profiles of antidiabetic and other drugs used in the 
elderly may change. Impaired renal and hepatic functions, reduced gastric acid secretion and gastric emptying, reduced splanchnic blood flow and absorptive capacity of the small intestine, reduction in liver mass and blood flow, changes in body composition, and increased sensitivity to drugs may lead to important changes in plasma concentrations. ${ }^{66,67}$

Premarketing clinical studies of rifapentine did not include sufficient numbers of subjects aged $\geq 65$ years to determine whether they respond differently from younger subjects. ${ }^{18}$ Other reported clinical experience, on the other hand, has not found differences in responses between the elderly and younger patients. ${ }^{68}$ According to the rifapentine's SmPC,

dose selection for an elderly patient should be cautious, usually starting at the low end of the dosing range, reflecting the greater frequency of decreased hepatic, renal, or cardiac function and of concomitant disease or other drug therapy. ${ }^{18}$

In elderly patients treated with rifampicin, the renal elimination of rifampicin was decreased proportionally to decrease of renal function; due to compensatory increase of liver elimination, the terminal half-life in serum was similar to that of younger patients. ${ }^{13}$ Nonetheless, the rifampicin's SmPC states that "caution should be exercised in using rifampicin in such patients, especially if there is evidence of impaired liver function". ${ }^{13}$ Although rifampicin and rifapentine share similar metabolic pathways, ${ }^{25,29}$ there are no pharmacokinetic studies performed so far that would explain the behavior of rifapentine in patients with impaired renal, hepatic, or cardiac function. These functions may be additionally compromised in the elderly with type 2 DM. Thus, the pharmacokinetic behavior of rifapentine in this population must be explored before its widespread use.

Bladder dysfunction in diabetes, often pathophysiologically overlapped with the physiological deterioration of bladder function in the elderly, should be evaluated when the use of rifamycins is considered because urination disorders, including infections, have been reported with this drug group. ${ }^{13,18}$ Rifapentine seems to have a slightly more advantageous urinary profile as compared to rifampicin; nonetheless, a synergistically induced increased risk of urinary disorders is most probably possible with both drugs when used in diabetes patients.

\section{Renal impairment}

About $30 \%-35 \%$ of patients with type $1 \mathrm{DM}$ and $10 \%-40 \%$ of those with type $2 \mathrm{DM}$ will at some point develop renal failure. ${ }^{69}$ Only about $17 \%$ of an administered dose of rifapentine is excreted via the kidneys, ${ }^{18,25}$ so renal impairment should not have a substantial influence on the blood levels of the drug. However, to date, the pharmacokinetics of rifapentine have not been evaluated in renal-impaired patients, and the clinical significance of impaired renal function on the disposition of rifapentine and its 25-desacetyl metabolite is not known. The SmPC of rifampicin, the renal excretion of which accounts for about one-third of the administered dose, states that "cautions should be taken in case of renal impairment if dose $>600 \mathrm{mg} /$ day". ${ }^{13}$ Following a single $900 \mathrm{mg}$ oral dose of rifampicin in patients with varying degrees of renal insufficiency, the mean half-life increased from $3.6 \mathrm{~h}$ in healthy adults to $5.0,7.3$, and $11.0 \mathrm{~h}$ in patients with glomerular filtration rates of $30-50 \mathrm{~mL} / \mathrm{min}$, in patients with $<30 \mathrm{~mL} / \mathrm{min}$, and in anuric patients, respectively. ${ }^{29}$ Moreover, acute renal failure usually due to acute tubular necrosis or acute interstitial nephritis has been reported with rifampicin, while with rifapentine no such effect has been observed. ${ }^{13,18}$ Due to a more favorable renal profile of rifapentine (a smaller proportion of renal excretion and no renal toxicity) than the one of rifampicin, rifapentine may be more suitable for use in patients with impaired renal function, including diabetes patients. This, however, has to be confirmed in pharmacokinetic studies.

Since rifapentine and related compounds excreted in urine account for only $17 \%$ of the administered dose, neither hemodialysis nor forced diuresis is expected to enhance the systemic elimination of unchanged rifapentine from the patient's circulation. ${ }^{18}$ While no pharmacokinetic data are available for rifapentine, the SmPC of rifampicin suggests that hemodialysis may cause a significant decrease in the blood levels of the drug. ${ }^{13}$ Woo et al found that patients with TB on maintenance dialysis had suboptimal blood concentrations of rifampicin, suggesting that treatment should be either with higher doses of the drug or with additional replacement doses given after each dialysis. ${ }^{70}$ On the other hand, Malone et al found that rifampicin was not significantly removed by hemodialysis. ${ }^{71}$ Overall, guides on the use of rifampicin in dialysis suggest that no rifampicin dose adjustments are required, ${ }^{13}$ but neither for this drug nor for rifapentine there are randomized controlled trials that provide evidence to guide TB treatment in renal failure/dialysis.

Diabetic kidney disease (DKD) associated with microor macroalbuminuria represents another significant challenge, especially in case of rifapentine ( $\sim 99 \%$ protein binding), although rifampicin is also highly protein-bound. Hypoalbuminemia, as a consequence of DKD or severe 
illness in TB patients, may lead to increased blood concentrations of the drug, an effect followed by dilution of drug's free fraction in an increased volume of distribution $(\mathrm{Vd})$ and rapid clearance. ${ }^{25}$

\section{Hepatic impairment}

Broad spectrum of liver disease (alteration of liver enzymes, nonalcoholic fatty liver disease, cirrhosis, hepatocellular carcinoma, acute liver failure) can be associated with type $2 \mathrm{DM}^{72}$ Type $1 \mathrm{DM}$ is not associated with hepatic fat accumulation if glycemia is well controlled, but type 2 DM may have a $70 \%$ correlation, regardless of glycemic control. ${ }^{72}$ Nonalcoholic fatty liver disease-associated alterations of liver function and transporter expression can lead to dramatic changes in drug disposition, whereas acute hepatic insufficiency influences drug's pharmacokinetics through changes in hepatic blood flow, liver enzyme activity, and the binding of drugs to plasma proteins. ${ }^{73,74}$

Following oral administration of a single $600 \mathrm{mg}$ dose of rifapentine to mild-to-severe hepatic impaired patients $(n=15)$, the PK parameters of rifapentine and 25-desacetyl metabolite were similar among patients with different levels of hepatic impairment. The obtained PK parameters were also similar to those observed in another study in healthy volunteers $(n=12) .{ }^{18}$ However, the SmPC of rifapentine concludes that the clinical significance of impaired hepatic function on the disposition of rifapentine and its 25-desacetyl metabolite is not known. ${ }^{18}$ The SmPC of rifampicin, on the other hand, warns that a daily dose of $8 \mathrm{mg} / \mathrm{kg}$ should not be exceeded in patients with impaired liver function. ${ }^{13}$ Competition between rifampicin and bilirubin for hepatic excretion may cause hyperbilirubinemia in the early days of treatment. ${ }^{13}$ Cases of hyperbilirubinemia have been observed in patients treated with rifapentine as well, but their pathogenesis and frequency still remain unclear.

The rifamycin class is associated with serious hepatic events, and patients with abnormal liver tests and/or liver disease, including diabetic patients, should only be given rifapentine in cases of necessity with caution and under strict medical supervision. ${ }^{18}$ In premarketing clinical studies, hypertransaminasemia was observed more frequently in the rifampicin than in the rifapentine group, ${ }^{13,18}$ but it is still not clear whether rifapentine is safer than rifampicin for use in liver failure.

\section{Adrenal insufficiency}

Patients with the impaired glucose tolerance induced by adrenal disorders may benefit more of the treatment with rifapentine than with rifampicin. Cases of adrenal insufficiency caused by rifampicin have been observed among patients with compromised adrenal function, but rifapentine does not seem to exert the same effect. ${ }^{13,18}$

\section{Metabolic imbalances}

Diabetes patients often present electrolyte disturbances, including hyperkalemia. In the diabetic with ketoacidosis, hyperkalemia is attributable to reduced renal function, acidosis, the release of potassium from cells due to glycogenolysis, and lack of insulin, whereas chronic hyperkalemia in diabetics is most often attributable to hyporeninemic hypoaldosteronism. ${ }^{75}$ The SmPC of rifapentine states that during both initial and continuation phases of clinical studies, cases of hyperkalemia have been observed in $\geq 1 \%$ of TB patients. ${ }^{18}$ Contrastingly, although there are reports of hyperkalemia in patients treated with rifampicin, it is always associated with previous adrenal insufficiency. ${ }^{13}$ Until more data are collected, special cautions should be taken when rifapentine is administered to diabetes patients with hyperkalemia or adrenal insufficiency.

Cases of both hyper- and hypoglycemia have been observed during clinical trials with rifapentine $(>1 \%){ }^{18}$ while the early use of rifampicin may be associated with hyperglycemia, as reported by several authors. ${ }^{76,77}$ Rifampicin is thought to boost glucose intestinal absorption leading to this effect. ${ }^{76,77}$ Although this adverse effect is not included in the SmPC of rifampicin, strict controls of glycemia may be required in patients receiving either of these drugs.

\section{Infections}

The greater frequency and severity of infections in diabetic patients is caused by the immune dysfunction, micro- and macro-angiopathies, neuropathy, decrease in the antibacterial activity of urine, and gastrointestinal and urinary dysmotility. ${ }^{10}$ In premarketing clinical trials with rifapentine, $>10 \%$ of treated patients reported urinary infections, which may represent an important downside of this drug's use in the diabetic population. ${ }^{18}$ Urinary tract infections have been reported with rifampicin as well, but again, its SmPC does not provide data on this side effect. Both antibiotics should be used with special caution in immunocompromised patients, including those with DM, due to increased risk of Clostridium difficile infection. ${ }^{13,18}$

\section{Adverse drug reactions Hepatic and gastrointestinal reactions}

Drugs from the rifamycin class are associated with serious hepatocellular damage, and it is generally accepted that 
patients with abnormal liver tests and/or liver disease should only be given these drugs in cases of necessity. Hypetransaminasemia was the most commonly observed hepatic event in premarketing clinical trials with rifapentine $(>1 \%)$, and it was slightly less frequent than that observed in studies with rifampicin. ${ }^{13,18}$ Lower doses and/or careful monitoring of liver function may be necessary when rifamycins are combined with thiazolidinediones and acarbose, also known for their hepatotoxic potential.

Dyspepsia, nausea, vomiting, constipation, and diarrhea have been observed in $>1 \%$ of the patients treated with rifapentine. ${ }^{18}$ Gastrointestinal side effects observed with rifampicin include nausea and dyspepsia ( $2 \%$ of patients), while heartburn, anorexia, vomiting, flatulence, cramps, and diarrhea occur less frequently. ${ }^{13}$ Biguanides, alpha-glucosidase inhibitors, bromocriptine, glinides, SGLT-2 inhibitors, insulin analogs, GLP-1 receptor agonists, and pramlintide may cause gastrointestinal disturbances as well; thus, clinicians must take this into consideration when treating diabetes patients with TB.

\section{Hematologic reactions}

In addition to nephropathy and neuropathy, certain oral antidiabetes drugs can increase the risk of developing anemia in diabetes patients. While rifapentine carries the risk of normocytic hypochromic anemia ( $>10 \%$ of treated patients), episodes of acute hemolytic anemia have been described with rifampicin. ${ }^{13,18}$ Therefore, combining either of these drugs with metformin and the thiazolidinediones, also known to cause anemia, may potentiate the risk of this side effect.

\section{Cardiovascular reactions}

Hypertension has been reported in $>1 \%$ of the patients treated with rifapentine. ${ }^{18}$ Patients treated concomitantly with this drug and insulin glargine should be monitored for hypertension, since glargine has been related to hypertensive episodes as well. ${ }^{55}$ In contrast, exposure to rifampicin has been associated with decreased blood pressure, especially when rifampicin dosages were administered intermittently. ${ }^{13}$ Bromocriptine is a hypoglycemic agent that causes hypotension in about one-third of treated patients: $;{ }^{78}$ thus, rifampicin may enhance the possibility of hypotension when combined with this drug.

\section{Neurological interactions}

Both rifampicin and rifapentine were associated with peripheral neuropathy in clinical trials. Although this side effect was rare $\left(<1 \%\right.$ of treated patients) ${ }^{13,18}$ diabetes patients should be monitored for it, especially those who achieved rapid improvement in glycemic control with detemir, shown to induce acute painful peripheral neuropathy in these circumstances. ${ }^{56}$

\section{Therapeutic drug monitoring}

In patients with $\mathrm{TB}$ and comorbidities such as $\mathrm{DM}$, the pharmacological profile of drugs changes owing to altered food intake, malabsorption, altered metabolism, drug-drug interactions, or adverse events. ${ }^{79,80}$ About $75 \%$ of diabetes patients were shown to have subtherapeutic levels of isoniazid or rifampicin or both at 2 weeks of anti-TB therapy. ${ }^{81}$ Nondiabetic TB patients show subtherapeutic isoniazid levels in $50 \%$ of the cases, and subtherapeutic rifampicin drug levels in $41 \%$ of the cases, according to Heysell et al..$^{82}$ Several studies showed that the AUC over $24 \mathrm{~h}$ in the steady state divided by the MIC (AUC/MIC ratio) is the best predictive pharmacokinetic/pharmacodynamic parameter for determination of the efficacy of rifampicin. ${ }^{83,84}$ Although its kinetics is essentially time-dependent, because of the presence of a post-antibiotic effect, it also has concentration-dependent features. ${ }^{83,84}$ Available data for rifapentine are scarce, but suggestive of $24 \mathrm{~h}$ AUC/MIC-dependent response as well. ${ }^{85}$ Therefore, in cases of rifampicin and rifapentine, therapeutic drug monitoring (TDM) has a potential to prevent subinhibitory drug concentrations, improve sputum conversion and diminish the slow response rates.

Although there is evidence that diabetes is associated with slow response and lower anti-TB drugs levels as compared to nondiabetes individuals, ${ }^{86}$ and that blood concentration of rifamycin shows substantial interindividual variability, ${ }^{87}$ TDM is currently recommended in TB treatment guidelines as optional. Obstacles to TDM implementation were summarized in a recent meta-analysis performed by Mota et al. ${ }^{88}$

Alkabab et al showed that TDM at 2 weeks after rifamycin treatment initiation in patients with diabetes shortened mean time to sputum culture conversion $(p=0.01)$ and increased rates of culture conversion by 2 months ( $p=0.04$ ), compared to diabetic patients who did not undergo TDM. ${ }^{89}$ However, TDM has not yet become standard in daily clinical practice. Further pharmacokinetic studies that would explore potential differences in bioavailability of these drugs in diabetes patients, as well as pharmacogenomic studies that would identify polymorphisms associated with different exposures and responses to rifamycin drug class, are urgently needed.

\section{Conclusion and future directions}

Expanding use of rifapentine heightens the clinicians' need for information relative to its efficacy and safety, especially 
in vulnerable populations such as diabetics. Overall, there is very little clinical evidence on rifapentine use, and in many cases, the characteristics of other drugs from the rifamycin class, mainly rifampicin, are simply attributed to rifapentine. However, differences between pharmacokinetic and safety profiles of these two drugs may lead to important contrasts with regard to their suitability for use in different populations. The complexity of anti-TB treatment in diabetes patients is reflected through pharmacokinetic alterations due to the infection itself and alterations in glucose metabolism, and through drug-drug interactions, but also in the peculiar characteristics of certain drugs, such as auto-induction and strong CYP450-inducing potential in case of rifampicin. Therefore, predicting pharmacokinetic behavior and efficacy of drugs used for TB treatment in diabetes patients is very hard. Rifapentine seems to be more suitable for use in diabetes patients with renal impairment, owing to the fact that it does not cause renal toxicity and it is eliminated via kidneys in smaller proportions than rifampicin. Nonetheless, hypoalbuminemia associated with DKD may interfere more with free blood fraction of rifapentine than with that of rifampicin. The number of patients aged $\geq 65$ years included in clinical trials with rifapentine was insufficient to determine whether they respond differently from younger subjects; thus, rifampicin may be safer for use in these cases. On the other hand, owing to ABCB1 induction and a higher degree of CYP450 induction as compared to rifapentine, rifampicin may more significantly diminish the efficacy of oral antidiabetics and cause hyperglycemia. At the moment, it seems that rifapentine and rifampicin have a very similar palette of adverse effects that could be potentiated by their concomitant use with oral antidiabetics. Unlike oral antidiabetics, GLP-1 receptor agonists, and pramlintide, insulin analogs do not seem to affect the metabolism of rifamycin drug class. While the scientific community is waiting for the results from pharmacokinetic studies with diabetes patients treated with both rifapentine and rifampicin, studies addressing the safety of metformin use in patients treated with rifapentine, and spontaneous and clinical trials- proceeding reports on rifapentine side effects, DM in TB patients treated with either rifapentine or rifampicin should be managed with insulin analogs. Blood glucose levels in diabetes patients treated with rifapentine/rifampicin and oral antidiabetics should be closely monitored, and doses of the drugs adjusted in accordance with the observed results. Therapeutic monitoring of rifapentine/rifampicin should be performed in all diabetes patients whenever possible. Due to a number of unknowns related to rifapentine's pharmacology and clinical effects in diabetes patients, its use should be carefully considered and closely supervised at all times.

\section{Disclosure}

The authors report no conflicts of interest in this work.

\section{References}

1. Baker MA, Lin HH, Chang HY, Murray MB. The risk of tuberculosis disease among persons with diabetes mellitus: a prospective cohort study. Clin Infect Dis. 2012;54(6):818-825.

2. Jeon CY, Murray MB, Baker MA. Managing tuberculosis in patients with diabetes mellitus: why we care and what we know. Expert Rev Anti Infect Ther. 2012;10(8):863-868.

3. Jiménez-Corona ME, Cruz-Hervert LP, García-García L, et al. Association of diabetes and tuberculosis: impact on treatment and posttreatment outcomes. Thorax. 2013;68(3):214-220.

4. Alisjahbana B, Sahiratmadja E, Nelwan EJ, et al. The effect of type 2 diabetes mellitus on the presentation and treatment response of pulmonary tuberculosis. Clin Infect Dis. 2007;45(4):428-435.

5. Stevenson CR, Forouhi NG, Roglic G, et al. Diabetes and tuberculosis: the impact of the diabetes epidemic on tuberculosis incidence. $B M C$ Public Health. 2007;7:234.

6. Lönnroth K, Roglic G, Harries AD. Improving tuberculosis prevention and care through addressing the global diabetes epidemic: from evidence to policy and practice. Lancet Diabetes Endocrinol. 2014; 2(9):730-739.

7. Lee MR, Huang YP, Kuo YT, et al. Diabetes mellitus and latent tuberculosis infection: a systemic review and metaanalysis. Clin Infect Dis. 2017;64(6):719-727.

8. Zumla AI, Gillespie SH, Hoelscher M, et al. New antituberculosis drugs, regimens, and adjunct therapies: needs, advances, and future prospects. Lancet Inf Dis. 2014;14(4):327-340.

9. Dostalek M, Akhlaghi F, Puzanovova M. Effect of diabetes mellitus on pharmacokinetic and pharmacodynamic properties of drugs. Clin Pharmacokinet. 2012;51(8):481-499.

10. Casqueiro J, Casqueiro J, Alves C. Infections in patients with diabetes mellitus: a review of pathogenesis. Indian J Endocrinol Metab. 2012;16(Suppl 1):S27-S36.

11. Restrepo BI. Diabetes and tuberculosis. Microbiol Spectr. 2016;4(6): TNM17-0023-2016

12. Isoniazid ${ }^{\circledR}$ (isoniazid) tablets [prescribing information]. London: Auden Mekenzie Limited; 2010.

13. Rifadin ${ }^{\mathbb{}}$ (rifampicin) hard capsules [prescribing information]. Guildford: Aventis Pharma Limited; 2005.

14. Sirturo ${ }^{\circledast}$ (bedaquiline) tablets [prescribing information]. Beerse: JanssenCilag Int. N.V.; 2012.

15. Deltyba ${ }^{\circledR}$ (delamanid) film-coated tablets [prescribing information]. Munchen: Otsuka Novel Products GmbH; 2014.

16. Gualano G, Capone S, Matteelli A, Palmieri F. New antituberculosis drugs: from clinical trial to programmatic use. Infect Dis Rep. 2016; 8(2):6569.

17. D'Ambrosio L, Centis R, Sotgiu G, Pontali E, Spanevello A, Migliori GB. New anti-tuberculosis drugs and regimens: 2015 update. ERJ Open Res. 2015;1(1):00010-2015.

18. Priftin $^{\circledR}$ (rifapentine) tablets [prescribing information]. Bridgewater, NJ: Sanofi-Aventis U.S. LLC; 1998.

19. WHO. Guidelines on the management of latent tuberculosis infection. 2015. Available from: http://apps.who.int/medicinedocs/documents/ s21682en/s21682en.pdf. Accessed May 6, 2017.

20. Huang YM, Zhao QP, Ren QM, Peng DL, Guo Y. The production and sales of anti-tuberculosis drugs in China. Infect Dis Poverty. 2016;5(1):88.

21. Cho SK, Yoon JS, Lee MG, et al. Rifampin enhances the glucoselowering effect of metformin and increases OCT1 mRNA levels in healthy participants. Clin Pharmacol Ther. 2011;89(3):416-421. 
22. Niemi M, Backman JT, Neuvonen M, Neuvonen PJ. Effect of rifampicin on the pharmacokinetics and pharmacodynamics of nateglinide in healthy subjects. Br J Clin Pharmacol. 2003;56(4):427-432.

23. Niemi M, Backman JT, Neuvonen M, Neuvonen PJ, Kivistö KT. Effects of rifampin on the pharmacokinetics and pharmacodynamics of glyburide and glipizide. Clin Pharmacol Ther. 2001;69(6):400-406.

24. Leow MK, Dalan R, Chee CB, et al. Latent tuberculosis in patients with diabetes mellitus: prevalence, progression and public health implications. Exp Clin Endocrinol Diabetes. 2014;122(9):528-532.

25. Drugbank.ca[homepage on the Internet]. DrugBank: Rifapentine. Available from: https://www.drugbank.ca/drugs/DB01201\#pharmacology. Accessed May 7, 2017.

26. Boeree MJ, Diacon AH, Dawson R, et al; PanACEA Consortium. A dose-ranging trial to optimize the dose of rifampin in the treatment of tuberculosis. Am J Respir Crit Care Med. 2015;191(9):1058-1065.

27. Savic RM, Lu Y, Bliven-Sizemore E, et al. Population pharmacokinetics of rifapentine and desacetyl rifapentine in healthy volunteers: nonlinearities in clearance and bioavailability. Antimicrob Agents Chemother. 2014;58(6):3035-3042.

28. Savic RM, Weiner M, MacKenzie WR, et al; Tuberculosis Trials Consortium of the Centers for Disease Control and Prevention. Defining the optimal dose of rifapentine for pulmonary tuberculosis: exposureresponse relations from two phase II clinical trials. Clin Pharmacol Ther. 2017;102(2):321-331

29. Drugbank.ca [homepage on the Internet]. DrugBank: Rifampicin. Available from: https://www.drugbank.ca/drugs/DB01045. Accessed May 7, 2017.

30. Nijland HM, Ruslami R, Stalenhoef JE, et al. Exposure to rifampicin is strongly reduced in patients with tuberculosis and type 2 diabetes. Clin Infect Dis. 2006;43(7):848-854.

31. Weiner M, Egelund EF, Engle M, et al. Pharmacokinetic interaction of rifapentine and raltegravir in healthy volunteers. J Antimicrob Chemother. 2014;69(4):1079-1085.

32. Temple ME, Nahata MC. Rifapentine: its role in the treatment of tuberculosis. Ann Pharmacother. 1999;33(11):1203-1210.

33. Podany AT, Bao Y, Swindells S, et al; AIDS Clinical Trials Group A5279 Study Team. Efavirenz pharmacokinetics and pharmacodynamics in HIV-infected persons receiving rifapentine and isoniazid for tuberculosis prevention. Clin Infect Dis. 2015;61(8):1322-1327.

34. Svensson EM, Murray S, Karlsson MO, Dooley KE. Rifampicin and rifapentine significantly reduce concentrations of bedaquiline, a new anti-TB drug. J Antimicrob Chemother. 2015;70(4):1106-1114.

35. Dooley KE, Bliven-Sizemore EE, Weiner M, et al. Safety and pharmacokinetics of escalating daily doses of the antituberculosis drug rifapentine in healthy volunteers. Clin Pharmacol Ther. 2012;91(5):881-888.

36. Holstein A, Beil W. Oral antidiabetic drug metabolism: pharmacogenomics and drug interactions. Expert Opin Drug Metab Toxicol. 2009;5(3):225-241.

37. Zheng HX, Huang Y, Frassetto LA, Benet LZ. Elucidating rifampin's inducing and inhibiting effects on glyburide pharmacokinetics and blood glucose in healthy volunteers: unmasking the differential effect of enzyme induction and transporter inhibition for a drug and its primary metabolite. Clin Pharmacol Ther. 2009;85(1):78-85.

38. Lin JH, Yamazaki M. Role of P-glycoprotein in pharmacokinetics: clinical implications. Clin Pharmacokinet. 2003;42(1):59-98.

39. Renton KW. Cytochrome P450 regulation and drug biotransformation during inflammation and infection. Curr Drug Metab. 2004;5(3): 235-243.

40. Aitken AE, Richardson TA, Morgan ET. Regulation of drug-metabolizing enzymes and transporters in inflammation. Annu Rev Pharmacol Toxicol. 2006;46:123-149.

41. Xu DX, Wang JP, Sun MF, Chen YH, Wei W. Lipopolysaccharide downregulates the expressions of intestinal pregnane $\mathrm{x}$ receptor and cytochrome p450 3a11. Eur J Pharmacol. 2006;536(1-2):162-170.

42. Cameron AR, Morrison VL, Levin D, et al. Anti-inflammatory effects of metformin irrespective of diabetes status. Circ Res. 2016;119(5): $652-665$.
43. Drugbank.ca [homepage on the Internet]. DrugBank: Metformin. Available from: https://www.drugbank.ca/drugs/DB00331. Accessed May 9, 2017.

44. Williamson B, Dooley KE, Zhang Y, Back DJ, Owen A. Induction of influx and efflux transporters and cytochrome p450 3a4 in primary human hepatocytes by rifampin, rifabutin, and rifapentine. Antimicrob Agents Chemother. 2013;57(12):6366-6369.

45. Niazi AK, Kalra S. Diabetes and tuberculosis: a review of the role of optimal glycemic control. J Diabetes Metab Disord. 2012;11(1):28.

46. Rodbard HW, Jellinger PS, Davidson JA, et al. Statement by an American Association of Clinical Endocrinologists/American College of Endocrinology consensus panel on type 2 diabetes mellitus: an algorithm for glycemic control. Endocr Pract. 2009;15(6):540-559.

47. Duckworth WC, Kitabchi AE. Insulin metabolism and degradation. Endocr Rev. 1981;2(2):210-233.

48. Ferrannini E, Wahren J, Faber OK, Felig P, Binder C, DeFronzo RA. Splanchnic and renal metabolism of insulin in human subjects: a doseresponse study. Am J Physiol. 1983;244(6):E517-E527.

49. Rabkin R, Ryan MP, Duckworth WC. The renal metabolism of insulin. Diabetologia. 1984;27(3):351-357.

50. Rabkin R, Simon NM, Steiner S, Colwell JA. Effect of renal disease on renal uptake and excretion of insulin in man. N Engl J Med. 1970; 282(4):182-187.

51. Evans M, Schumm-Draeger PM, Vora J, King AB. A review of modern insulin analogue pharmacokinetic and pharmacodynamic profiles in type 2 diabetes: improvements and limitations. Diabetes Obes Metab. 2011;13(8):677-684.

52. Humalog ${ }^{\circledR}$ (insulin lispro) injection [prescribing information]. Indianapolis, IN: Eli Lilly and Company; 2011.

53. NovoLog ${ }^{\circledR}$ (insulin aspart) injection [prescribing information]. Princeton, NJ: Novo Nordisk Inc; 2011.

54. Apidra ${ }^{\circledR}$ (insulin glulisine) injection [prescribing information]. Bridgewater, NJ: Sanofi-Aventis; 2009.

55. Lantus ${ }^{\circledR}$ (insulin glargine) injection [prescribing information]. Bridgewater, NJ: Sanofi-Aventis; 2007.

56. Levemir $^{\circledR}$ (insulin detemir) injection [prescribing information]. Bagsvaerd, Denmark: Novo Nordisk Inc; 2015.

57. Tresiba ${ }^{\circledR}$ (insulin degludec) injection [prescribing information]. Bagsvaerd, Denmark: Novo Nordisk Inc; 2013

58. Saxenda ${ }^{\circledR}$ (liraglutide) injection [prescribing information]. Bagsvaerd, Denmark: Novo Nordisk Inc; 2015.

59. SymlinPen ${ }^{\circledR}$ (pramlintide acetate) injection [prescribing information]. London: AstraZeneca; 2015.

60. Bemer-Melchior P, Bryskier A, Drugeon HB. Comparison of the in vitro activities of rifapentine and rifampicin against Mycobacterium tuberculosis complex. J Antimicrob Chemother. 2000;46(4):571-576.

61. Colhoun HM, Betteridge DJ, Durrington PN, et al; CARDS investigators. Primary prevention of cardiovascular disease with atorvastatin in type 2 diabetes in the Collaborative Atorvastatin Diabetes Study (CARDS): multicentre randomised placebo-controlled trial. Lancet. 2004;364(9435):685-696.

62. Skottheim IB, Gedde-Dahl A, Hejazifar S, Hoel K, Asberg A. Statin induced myotoxicity: the lactone forms are more potent than the acid forms in human skeletal muscle cells in vitro. Eur J Pharm Sci. 2008; 33(4-5):317-325.

63. Marques MP, Coelho EB, Dos Santos NA, Geleilete TJ, Lanchote VL. Dynamic and kinetic disposition of nisoldipine enantiomers in hypertensive patients presenting with type-2 diabetes mellitus. Eur J Clin Pharmacol. 2002;58(9):607-614.

64. Williams DL, Spring L, Collins L, et al. Contribution of rpoB mutations to development of rifamycin cross-resistance in Mycobacterium tuberculosis. Antimicrob Agents Chemother. 1998;42(7):1853-1857.

65. Duanmu HJ, Liu YH, Jiang GL, Wang SM, Fu YH. In vitro study on cross resistance of rifampin and rifapentine for Mycobacterium tuberculosis. Zhonghua Jie He He Hu Xi Za Zhi. 2005;28(3):192-194.

66. Corazza GR, Frazzoni M, Gatto MR, Gasbarrini G. Ageing and smallbowel mucosa: a morphometric study. Gerontology. 1986;32(1):60-65. 
67. Fülöp T Jr, Wórum I, Csongor J, Fóris G, Leövey A. Body composition in elderly people. I. Determination of body composition by multiisotope method and the elimination kinetics of these isotopes in healthy elderly subjects. Gerontology. 1985;31(1):6-14.

68. Keung AC, Eller MG, Weir SJ. Single-dose pharmacokinetics of rifapentine in elderly men. Pharm Res. 1998;15(8):1286-1291.

69. Cade WT. Diabetes-related microvascular and macrovascular diseases in the physical therapy setting. Phys Ther. 2008;88(11):1322-1335.

70. Woo J, Leung A, Chan K, Lai KN, Teoh R. Pyrazinamide and rifampicin regimens for patients on maintenance dialysis. Int J Artif Organs. 1988;11(3):181-185.

71. Malone RS, Fish DN, Spiegel DM, Childs JM, Peloquin CA. The effect of hemodialysis on isoniazid, rifampin, pyrazinamide, and ethambutol. Am J Respir Crit Care Med. 1999;159(5 Pt 1):1580-1584.

72. Tolman KG, Fonseca V, Dalpiaz A, Tan MH. Spectrum of liver disease in type 2 diabetes and management of patients with diabetes and liver disease. Diabetes Care. 2007;30(3):734-743.

73. Merrell MD, Cherrington NJ. Drug metabolism alterations in nonalcoholic fatty liver disease. Drug Metab Rev. 2011;43(3):317-334.

74. Rodighiero V. Effects of liver disease on pharmacokinetics. An update. Clin Pharmacokinet. 1999;37(5):399-431.

75. Liamis G, Liberopoulos E, Barkas F, Elisaf M. Diabetes mellitus and electrolyte disorders. World J Clin Cases. 2014;2(10):488-496.

76. Takasu N, Yamada T, Miura H, et al. Rifampicin-induced early phase hyperglycemia in humans. Am Rev Respir Dis. 1982;125(1):23-27.

77. Atkin SL, Masson EA, Bodmer CW, Walker BA, White MC. Increased insulin requirement in a patient with Type 1 diabetes on rifampicin. Diabet Med. 1993;10(4):392.

78. Bromocriptine ${ }^{\circledR}$ (bromocriptine mesylate) tablets [prescribing information]. Takeley: Meda Pharmaceuticals; 2012.

79. Alsultan A, Peloquin CA. Therapeutic drug monitoring in the treatment of tuberculosis: an update. Drugs. 2014;74(8):839-854.

80. Peloquin CA. Therapeutic drug monitoring in the treatment of tuberculosis. Drugs. 2002;62(15):2169-2183.
81. Babalik A, Ulus IH, Bakirci N, et al. Plasma concentrations of isoniazid and rifampin are decreased in adult pulmonary tuberculosis patients with diabetes mellitus. Antimicrob Agents Chemother. 2013;57(11): 5740-5742.

82. Heysell SK, Moore JL, Staley D, Dodge D, Houpt ER. Early therapeutic drug monitoring for isoniazid and rifampin among diabetics with newly diagnosed tuberculosis in Virginia, USA. Tuberc Res Treat. 2013; 2013:129723.

83. Gumbo T, Louie A, Deziel MR, et al. Concentration-dependent mycobacterium tuberculosis killing and prevention of resistance by rifampin. Antimicrob Agents Chemother. 2007;51(11):3781-3788.

84. Jayaram R, Gaonkar S, Kaur P, etal. Pharmacokinetics-pharmacodynamics of rifampin in an aerosol infection model of tuberculosis. Antimicrob Agents Chemother. 2003;47(7):2118-2124.

85. Dooley K, Flexner C, Hackman J, et al. Repeated administration of high-dose intermittent rifapentine reduces rifapentine and moxifloxacin plasma concentrations. Antimicrob Agents Chemother. 2008; 52(11):4037-4042.

86. Heysell SK, Moore JL, Keller SJ, Houpt ER. Therapeutic drug monitoring for slow response to tuberculosis treatment in a state control program, Virginia, USA. Emerg Infect Dis. 2010;16(10):1546-1553.

87. Kwara A, Cao L, Yang H, et al. Factors associated with variability in rifampin plasma pharmacokinetics and the relationship between rifampin concentrations and induction of efavirenz clearance. Pharmacotherapy. 2014;34(3):265-271.

88. Mota L, Al-Efraij K, Campbell JR, Cook VJ, Marra F, Johnston J. Therapeutic drug monitoring in anti-tuberculosis treatment: a systematic review and meta-analysis. Int J Tuberc Lung Dis. 2016;20(6): 819-826.

89. Alkabab Y, Keller S, Dodge D, Houpt E, Staley D, Heysell S. Early interventions for diabetes related tuberculosis associate with hastened sputum microbiological clearance in Virginia, USA. BMC Infect Dis. 2017;17(1):125.
Drug Design, Development and Therapy

\section{Publish your work in this journal}

Drug Design, Development and Therapy is an international, peerreviewed open-access journal that spans the spectrum of drug design and development through to clinical applications. Clinical outcomes, patient safety, and programs for the development and effective, safe, and sustained use of medicines are the features of the journal, which

\section{Dovepress}

has also been accepted for indexing on PubMed Central. The manuscript management system is completely online and includes a very quick and fair peer-review system, which is all easy to use. Visit http://www.dovepress.com/testimonials.php to read real quotes from published authors. 